\title{
OPEN Incidence of malignancy and related mortality after kidney transplantation: a nationwide, population-based cohort study in Korea
}

Seri Jeong ${ }^{1}$, Ho Sup Lee ${ }^{2}$, Seom Gim Kong ${ }^{3}$, Da Jung Kim² ${ }^{2}$ Sangjin Lee ${ }^{4}$, Min-Jeong Park ${ }^{1}$, Wonkeun Song ${ }^{1}$, John Hoon Rim $^{5,6}$ \& Hyung Jik Kim ${ }^{7 凶}$

Post-transplant malignancy (PTM) is a leading cause of premature mortality among kidney transplantation recipients. However, population-based cohort studies that cover incidence, mortality, and risk factors for PTM are rarely reported, especially in East Asia. We designed a retrospective cohort study using a national population-based database. A total of 9915 kidney recipients between 2003 and 2016 were included. During this period, 598 cases $(6.0 \%)$ of de novo PTM occurred. The most common PTM was thyroid cancer (14.2\%), followed by colorectal (11.2\%), kidney (10.7\%), and stomach cancers (8.9\%). The standardised incidence ratio for all-site cancer was 3.9. The risks of Kaposi sarcoma (192.9) and kidney cancer (21.1) were more than 10 times those of the general population. Cancerrelated deaths were $89(14.9 \%)$ with liver cancer being the highest (14.6\%), followed by lung cancer (13.5\%), non-Hodgkin lymphoma (NHL) (12.4\%), stomach cancer $(9.0 \%)$, and colorectal cancer $(7.9 \%)$. The standardised mortality ratio (SMR) was slightly elevated (1.4). A notable increase in SMR was observed for lymphoma (9.3 for Hodgkin lymphoma and 5.5 for NHL). Older age and graft failure were significantly related to PTM. These findings reflecting geographical variation have implications for the development of strategies for fatal cancers to prevent premature deaths from PTM.

Post-transplant malignancy (PTM) is a devastating complication of kidney transplantation and the second most common cause of mortality in kidney recipients ${ }^{1}$. In general, kidney transplant recipients have a higher incidence of malignancies compared with the general population ${ }^{1,2}$. Poorer outcomes, including mortality and graft failure, have been reported in patients with $\mathrm{PTM}^{2-5}$. However, the incidence, mortality, and risk factors for PTM show considerable ethnic and geographic variation. Non-melanoma skin cancer (NMSC) and non-Hodgkin lymphoma (NHL) are the most common PTMs in the United States and Europe $\mathrm{e}^{6-9}$. In contrast, stomach and kidney cancers are commonly reported PTMs in Asian countries ${ }^{10-12}$.

There have only been a few reports of PTM in South Korea. Hwang et al. ${ }^{11}$ presented stomach cancer and malignant lymphoma as the most common PTMs based on a 40-year single-centre dataset. Most previous studies are derived from single-centre data with a relatively small number of recipients ${ }^{13-15}$. Recently Heo et al. ${ }^{16}$ and Park et al. ${ }^{17}$ found thyroid cancer to be the most common PTMs identified in from 5- and 8-year nationwide databases, respectively. The relatively short mean duration of follow-up may affect the accuracy of data concerning late-onset PTM. Furthermore, mortality data derived from PTM and risk factors for PTM were limited in the previous studies. Reports from Western countries have yielded contradictory results on cancer mortality; risk

${ }^{1}$ Department of Laboratory Medicine, Kangnam Sacred Heart Hospital, Hallym University College of Medicine, Seoul 07441, South Korea. 'Department of Hematology-Oncology, Kosin University College of Medicine, Busan 49267, South Korea. ${ }^{3}$ Department of Pediatrics, Kosin University College of Medicine, Busan 49267, South Korea. "Department of Statistics, Graduate School, Pusan National University, Busan 46241, South Korea. ${ }^{5}$ Department of Pharmacology, Yonsei University College of Medicine, Seoul 03722, South Korea. ${ }^{6}$ Department of Medicine, Physician-Scientist Program, Yonsei University Graduate School of Medicine, Seoul 03722, South Korea. ${ }^{7}$ Department of Internal Medicine, Hallym University Sacred Heart Hospital, Hallym University College of Medicine, 22, Gwanpyeong-ro 170 beon-gil, Dongan-gu, 14068 Anyang-si, Gyeonggi-do, South Korea. ${ }^{\circledR}$ email: hyung@hallym.or.kr 


\begin{tabular}{|c|c|c|c|c|}
\hline Variable ${ }^{*}$ & Total patients & PTM & Non-PTM & $P$-value \\
\hline \multicolumn{5}{|l|}{ Age, years } \\
\hline$<40$ & $3225(32.5)$ & $154(25.8)$ & $3071(33.0)$ & \multirow{3}{*}{0.001} \\
\hline $40-59$ & $5736(57.9)$ & $377(63.0)$ & $5359(57.5)$ & \\
\hline$>59$ & $954(9.6)$ & $67(11.2)$ & $887(9.5)$ & \\
\hline \multicolumn{5}{|l|}{ Sex } \\
\hline Male & $5970(60.2)$ & $341(57.0)$ & $5629(60.4)$ & \multirow{2}{*}{0.110} \\
\hline Female & $3945(39.8)$ & $257(43.0)$ & $3688(39.6)$ & \\
\hline Total person-years & 53,835 & 2809 & 51,026 & \\
\hline Male & 32,175 & 1619 & 30,556 & \\
\hline Female & 21,660 & 1190 & 20,470 & \\
\hline \multicolumn{5}{|l|}{ Induction immunosuppressant } \\
\hline Basiliximab & $1135(11.4)$ & $47(7.9)$ & $1088(11.7)$ & 0.004 \\
\hline Anti-thymocyte globulin & $7821(78.9)$ & $442(73.9)$ & $7379(79.2)$ & 0.002 \\
\hline \multicolumn{5}{|l|}{ Maintenance immunosuppressant } \\
\hline Tacrolimus + MMF + corticosteroid & $6958(70.2)$ & $355(59.4)$ & $6603(70.9)$ & $<0.001$ \\
\hline Cyclosporine +MMF + corticosteroid & $2158(21.8)$ & $210(35.1)$ & $1948(20.9)$ & $<0.001$ \\
\hline \multicolumn{5}{|l|}{ Infection } \\
\hline CMV disease & $1018(10.3)$ & $61(10.2)$ & $957(10.3)$ & 0.956 \\
\hline EBV mononucleosis & $11(0.1)$ & $1(0.2)$ & $10(0.1)$ & 0.670 \\
\hline Graft failure & $442(4.5)$ & $63(10.5)$ & 408 (4.4) & $<0.001$ \\
\hline \multicolumn{5}{|l|}{ Year of transplant } \\
\hline $2003-2009$ & $3473(35.0)$ & $383(64.1)$ & $3090(33.2)$ & $<0.001$ \\
\hline $2010-2016$ & $6442(65.0)$ & $215(36.0)$ & $6227(66.8)$ & \\
\hline
\end{tabular}

Table 1. Comparison of characteristics between patients with post-transplant malignancies (PTM) versus without PTM. CMV cytomegalovirus, EBV Epstein-Barr virus, MMF mycophenolate mofetil, PTM posttransplant malignancy. ${ }^{\star}$ Data are expressed as number and percentage.

of death in USA transplant recipients was not increased ${ }^{18}$, but those in Canada ${ }^{3}$, Australia, and New Zealand ${ }^{19}$ were three times higher than the general population. A large population-based study with a lengthy follow-up is essential in order to understand PTM and establish cancer surveillance strategies for kidney recipients.

The present study was based on the use of a comprehensive database operated by the National Health Insurance (NHI) of the Korean government. This database contains all the records of healthcare utilisation by kidney recipients who were enrolled in the Rare Intractable Disease (RID) system for reimbursement purposes. Registration in such is confirmed by a certified physician based on the RID criteria, which reflect international guidelines, and is verified by the NHI system. Therefore, the use of this database was suitable for the investigation of PTM among kidney recipients.

Using this database, we performed a comprehensive nationwide population-based analysis with the permitted maximum lengthy follow-up duration to investigate the incidence, mortality, and risk factors for PTM. This study can facilitate prevention and management of PTMs, which will contribute to outcome improvements in kidney recipients.

\section{Results}

Characteristics of patients. A total of 9915 patients who underwent kidney transplantation from 2003 to 2016 were included in our study cohort. The median follow-up duration was 4.87 years, representing 53,835 person-years of follow-up. During this period, 598 cases of de novo PTM occurred. The baseline characteristics of these patients are presented in Table 1. Patients with PTM were older than the non-PTM group (median age: 48 vs. 46 years, $P<0.001$ ). There were significant differences between the PTM and non-PTM groups for immunosuppressive agents: induction ( $P=0.004$ for basiliximab, and $P=0.002$ for anti-thymocyte globulin), and maintenance $(P<0.001$ for calcineurin inhibitors, mycophenolate mofetil, and corticosteroid). There was a higher proportion of recipients who suffered from graft failure in the PTM group than the non-PTM group $(P<0.001)$. The proportion of PTM cases decreased from 383 (64.1\%) in 2003-2009 to 215 (36.03\%) in 2010-2016.

Incidence of PTM. The overall incidence of PTM was 6.0\% (598/9915). The cancer types and characteristics of PTMs are presented in Table 2. The most common PTM was thyroid cancer $(\mathrm{n}=85,14.2 \%)$, followed by colorectal cancer $(n=67,11.2 \%)$, kidney cancer $(n=64,10.7 \%)$, stomach cancer $(n=53,8.9 \%)$, and prostate cancer $(\mathrm{n}=49,8.2 \%)$. The median age of patients at diagnosis of PTM was 52.0 years (interquartile range: 16.0 years). Melanoma ( 40.0 years), cervical cancer (44.5 years), and oral cavity cancer ( 47.0 years) occurred in younger recipients, who were also relatively younger at the time of kidney transplantation. In contrast, oesophageal cancer (65.0 years), pancreatic cancer (60.0 years), ureter cancer (59.5 years), and NMSC (59.0 years) developed in older recipients, resulting in relatively longer duration from transplantation to PTM. The median interval 


\begin{tabular}{|c|c|c|c|c|c|c|c|c|c|c|c|c|}
\hline \multirow{2}{*}{$\begin{array}{l}\text { Type of } \\
\text { cancer }\end{array}$} & \multicolumn{3}{|c|}{ Number of cancer (\%) } & \multicolumn{3}{|c|}{ Age at transplant, years* } & \multicolumn{3}{|c|}{ Age at diagnosis of PTM, years* } & \multicolumn{3}{|c|}{$\begin{array}{l}\text { Time between transplant and PTM, } \\
\text { years* }\end{array}$} \\
\hline & Male & Female & Total & Male & Female & Total & Male & Female & Total & Male & \begin{tabular}{|l|l} 
Female \\
\end{tabular} & Total \\
\hline Thyroid & $30(8.8)$ & $55(21.4)$ & $85(14.2)$ & $\begin{array}{l}44.5 \\
(38.8-54.8)\end{array}$ & $\begin{array}{l}44.0 \\
(36.0-50.0)\end{array}$ & $\begin{array}{l}44.0 \\
(37.0-52.0)\end{array}$ & $\begin{array}{l}48.5 \\
(43.0-56.8)\end{array}$ & $\begin{array}{l}46.0 \\
(41.0-53.0)\end{array}$ & $\begin{array}{l}48.0 \\
(42.0-55.0)\end{array}$ & $2.7(1.8-6.5)$ & $4.0(2.1-6.6)$ & $3.4(1.8-6.5)$ \\
\hline Colorectal & $34(10.0)$ & $33(12.8)$ & $67(11.2)$ & $\begin{array}{l}46.5 \\
(39.3-56.5)\end{array}$ & $\begin{array}{l}47.0 \\
(34.0-54.0)\end{array}$ & \begin{tabular}{|l|}
47.0 \\
$(36.5-55.0)$
\end{tabular} & \begin{tabular}{|l|}
52.5 \\
$(45.3-59.0)$
\end{tabular} & $\begin{array}{l}51.0 \\
(38.0-59.0)\end{array}$ & $\begin{array}{l}52.0 \\
(42.0-59.0)\end{array}$ & $3.9(2.3-8.2)$ & $4.5(2.2-8.5)$ & $4.4(2.2-8.4)$ \\
\hline Kidney & $46(13.5)$ & $18(7.0)$ & $64(10.7)$ & $\begin{array}{l}44.0 \\
(37.3-52.8)\end{array}$ & $\begin{array}{l}45.0 \\
(36.5-53.0)\end{array}$ & \begin{tabular}{|l|}
45.0 \\
$(36.8-53.0)$
\end{tabular} & \begin{tabular}{|l|}
51.0 \\
$(41.3-59.0)$
\end{tabular} & $\begin{array}{l}53.0 \\
(43.3-58.3)\end{array}$ & $\begin{array}{l}51.5 \\
(41.8-59.0)\end{array}$ & $6.1(2.4-9.1)$ & $3.6(2.1-8.2)$ & $5.8(2.3-9.0)$ \\
\hline Stomach & $31(9.1)$ & $22(8.6)$ & $53(8.9)$ & $\begin{array}{l}52.0 \\
(47.0-55.5)\end{array}$ & $\begin{array}{l}52.0 \\
(46.0-58.0)\end{array}$ & \begin{tabular}{|l|}
52.0 \\
$(46.0-58.0)$
\end{tabular} & $\begin{array}{l}57.0 \\
(49.5-62.0)\end{array}$ & $\begin{array}{l}56.0 \\
(51.3-62.0)\end{array}$ & $\begin{array}{l}57.0 \\
(51.0-62.0)\end{array}$ & $5.2(3.3-7.0)$ & $4.8(2.7-6.5)$ & $5.0(3.3-6.8)$ \\
\hline Prostate & 49 (14.4) & $0(0.0)$ & $49(8.2)$ & $\begin{array}{l}52.0 \\
(45.0-58.0)\end{array}$ & - & $\begin{array}{l}52.0 \\
(45.0-58.0)\end{array}$ & \begin{tabular}{|l|}
55.0 \\
$(49.0-60.0)$
\end{tabular} & - & $\begin{array}{l}55.0 \\
(49.0-60.0)\end{array}$ & $3.6(1.3-6.4)$ & - & $3.6(1.3-6.4)$ \\
\hline Liver & $32(9.4)$ & $10(3.9)$ & $42(7.0)$ & $\begin{array}{l}51.0 \\
(45.8-55.0)\end{array}$ & $\begin{array}{l}49.5 \\
(31.5-51.5)\end{array}$ & $\begin{array}{l}49.5 \\
(40.3-54.8)\end{array}$ & \begin{tabular}{|l|}
54.5 \\
$(47.0-59.0)$
\end{tabular} & $\begin{array}{l}45.5 \\
(35.0-56.0)\end{array}$ & $\begin{array}{l}53.5 \\
(45.3-58.8)\end{array}$ & $3.3(1.7-6.0)$ & $2.7(1.8-4.6)$ & $3.2(1.8-5.7)$ \\
\hline Lung & $31(9.1)$ & $11(4.3)$ & $42(7.0)$ & $\begin{array}{l}54.0 \\
(45.5-59.0)\end{array}$ & $\begin{array}{l}53.5 \\
(41.0-55.0)\end{array}$ & \begin{tabular}{|l|}
53.5 \\
$(42.8-58.0)$
\end{tabular} & \begin{tabular}{|l|}
59.0 \\
$(49.0-63.5)$
\end{tabular} & $\begin{array}{l}53.0 \\
(44.0-58.5)\end{array}$ & $\begin{array}{l}58.0 \\
(47.3-62.8)\end{array}$ & $4.3(2.7-6.0)$ & $3.8(1.0-5.3)$ & $4.2(1.9-5.5)$ \\
\hline Breast & $1(0.3)$ & $35(13.6)$ & $36(6.0)$ & $\begin{array}{l}51.0 \\
(51.0-51.0)\end{array}$ & $\begin{array}{l}44.0 \\
(39.5-50.0)\end{array}$ & $\begin{array}{l}44.0 \\
(39.8-50.3)\end{array}$ & \begin{tabular}{|l|}
57.0 \\
$(57.0-57.0)$
\end{tabular} & $\begin{array}{l}48.0 \\
(44.5-53.0)\end{array}$ & $\begin{array}{l}48.0 \\
(44.8-53.8)\end{array}$ & $6.3(6.3-6.3)$ & $4.1(2.2-5.8)$ & $4.2(2.3-6.0)$ \\
\hline $\begin{array}{l}\text { Non- } \\
\text { Hodgkin } \\
\text { lymphoma }\end{array}$ & $13(3.8)$ & $14(5.4)$ & $27(4.5)$ & $\begin{array}{l}32.0 \\
(29.0-47.0)\end{array}$ & $\begin{array}{l}45.0 \\
(39.8-52.0)\end{array}$ & $\begin{array}{l}45.0 \\
(31.5-51.0)\end{array}$ & $\begin{array}{l}38.0 \\
(35.0-49.0)\end{array}$ & $\begin{array}{l}53.0 \\
(41.8-57.3)\end{array}$ & $\begin{array}{l}49.0 \\
(37.0-55.0)\end{array}$ & $3.3(1.6-9.0)$ & $5.3(2.4-7.1)$ & $5.1(1.7-7.8)$ \\
\hline $\begin{array}{l}\text { Urinary } \\
\text { bladder }\end{array}$ & $13(3.8)$ & $7(2.7)$ & $20(3.3)$ & $\begin{array}{l}52.0 \\
(39.0-55.0)\end{array}$ & $\begin{array}{l}48.5 \\
(41.5-51.0)\end{array}$ & $\begin{array}{l}48.5 \\
(38.8-54.3)\end{array}$ & $\begin{array}{l}54.0 \\
(48.0-57.0)\end{array}$ & $\begin{array}{l}51.0 \\
(48.0-54.5)\end{array}$ & $\begin{array}{l}53.5 \\
(47.8-57.0)\end{array}$ & $5.8(2.5-8.7)$ & $5.3(2.7-6.2)$ & $5.4(2.4-8.1)$ \\
\hline Cervix & $0(0.0)$ & $14(5.4)$ & $14(2.3)$ & - & $\begin{array}{l}39.5 \\
(37.0-45.3)\end{array}$ & $\begin{array}{l}39.5 \\
(37.0-45.3)\end{array}$ & - & \begin{tabular}{|l|}
44.5 \\
$(41.0-49.8)$
\end{tabular} & $\begin{array}{l}44.5 \\
(41.0-49.8)\end{array}$ & - & $3.8(2.3-6.0)$ & $3.8(2.3-6.0)$ \\
\hline $\begin{array}{l}\text { Non-mela- } \\
\text { noma skin }\end{array}$ & $9(2.6)$ & $4(1.6)$ & $13(2.2)$ & $\begin{array}{l}54.0 \\
(44.0-59.0)\end{array}$ & $\begin{array}{l}54.0 \\
(49.8-58.0)\end{array}$ & \begin{tabular}{|l|}
54.0 \\
$(44.0-59.0)$
\end{tabular} & $\begin{array}{l}61.0 \\
(52.0-66.0)\end{array}$ & $\begin{array}{l}56.5 \\
(53.5-62.3)\end{array}$ & $\begin{array}{l}59.0 \\
(52.0-66.0)\end{array}$ & $7.4(4.3-8.9)$ & $5.1(4.1-6.6)$ & $7.2(4.3-8.9)$ \\
\hline Oral cavity & $9(2.6)$ & $4(1.6)$ & $13(2.2)$ & \begin{tabular}{|l|}
46.0 \\
$(40.0-48.0)$ \\
\end{tabular} & $\begin{array}{l}46.0 \\
(36.0-41.5) \\
\end{array}$ & \begin{tabular}{|l|}
46.0 \\
$(37.0-48.0)$ \\
\end{tabular} & \begin{tabular}{|l|}
50.0 \\
$(41.0-53.0)$ \\
\end{tabular} & $\begin{array}{l}41.0 \\
(39.3-43.5) \\
\end{array}$ & $\begin{array}{l}47.0 \\
(41.0-51.0) \\
\end{array}$ & $2.8(1.9-4.9)$ & $3.1(1.7-4.6)$ & $2.8(1.8-4.9)$ \\
\hline Pancreas & $9(2.6)$ & $2(0.8)$ & $11(1.8)$ & \begin{tabular}{|l|}
57.0 \\
$(54.0-61.0)$
\end{tabular} & $\begin{array}{l}57.0 \\
(37.5-54.5)\end{array}$ & \begin{tabular}{|l|}
57.0 \\
$(53.5-62.0)$
\end{tabular} & $\begin{array}{l}60.0 \\
(56.0-61.0)\end{array}$ & $\begin{array}{l}50.0 \\
(42.0-58.0)\end{array}$ & $\begin{array}{l}60.0 \\
(54.5-63.5)\end{array}$ & $2.8(0.9-3.5)$ & $4.3(3.6-4.9)$ & $2.9(1.0-3.6)$ \\
\hline Leukaemia & $6(1.8)$ & $5(1.9)$ & $11(1.8)$ & $\begin{array}{l}47.0 \\
(41.0-50.8)\end{array}$ & $\begin{array}{l}47.0 \\
(33.0-48.0)\end{array}$ & \begin{tabular}{|l|}
47.0 \\
$(36.0-50.0)$
\end{tabular} & \begin{tabular}{|l|}
50.0 \\
$(44.3-54.3)$
\end{tabular} & $\begin{array}{l}46.0 \\
(37.0-53.0)\end{array}$ & $\begin{array}{l}48.0 \\
(40.0-53.5)\end{array}$ & $3.7(2.3-5.5)$ & $4.4(1.6-6.7)$ & $4.2(1.8-6.3)$ \\
\hline $\begin{array}{l}\text { Nasopharyn- } \\
\text { geal }\end{array}$ & $8(2.3)$ & $2(0.8)$ & $10(1.7)$ & $\begin{array}{l}49.5 \\
(47.0-53.0)\end{array}$ & $\begin{array}{l}49.0 \\
(48.3-48.8)\end{array}$ & \begin{tabular}{|l|}
49.0 \\
$(48.3-52.3)$
\end{tabular} & \begin{tabular}{|l|}
53.0 \\
$(49.0-57.3)$
\end{tabular} & $\begin{array}{l}51.0 \\
(50.0-52.0)\end{array}$ & $\begin{array}{l}52.5 \\
(49.5-56.3)\end{array}$ & $3.5(2.4-5.8)$ & $3.2(2.4-4.1)$ & $3.5(1.8-5.0)$ \\
\hline $\begin{array}{l}\text { Kaposi } \\
\text { sarcoma }\end{array}$ & $8(2.3)$ & $1(0.4)$ & $9(1.5)$ & $\begin{array}{l}42.5 \\
(33.0-55.5)\end{array}$ & $\begin{array}{l}46.0 \\
(63.0-63.0)\end{array}$ & $\begin{array}{l}46.0 \\
(34.0-57.0)\end{array}$ & $\begin{array}{l}47.0 \\
(34.0-57.0)\end{array}$ & $\begin{array}{l}65.0 \\
(65.0-65.0)\end{array}$ & $\begin{array}{l}55.0 \\
(35.0-60.0) \\
\end{array}$ & $2.2(1.1-5.0)$ & $2.4(2.4-2.4)$ & $2.4(1.1-4.2)$ \\
\hline Ovarian & $0(0.0)$ & $8(3.1)$ & $8(1.3)$ & - & $\begin{array}{l}44.5 \\
(40.0-50.8)\end{array}$ & $\begin{array}{l}44.5 \\
(40.0-50.8)\end{array}$ & - & $\begin{array}{l}50.5 \\
(43.8-53.8)\end{array}$ & $\begin{array}{l}50.5 \\
(43.8-53.8)\end{array}$ & - & $3.5(2.9-5.2)$ & $3.5(2.9-5.2)$ \\
\hline Uterus & $0(0.0)$ & $7(2.7)$ & $7(1.2)$ & - & $\begin{array}{l}52.0 \\
(36.0-55.5)\end{array}$ & $\begin{array}{l}52.0 \\
(36.0-55.5)\end{array}$ & - & $\begin{array}{l}55.0 \\
(42.5-56.0)\end{array}$ & $\begin{array}{l}55.0 \\
(42.5-56.0)\end{array}$ & - & $4.7(1.9-7.0)$ & $4.7(1.9-7.0)$ \\
\hline Gallbladder & $5(1.5)$ & $1(0.4)$ & $6(1.0)$ & $\begin{array}{l}56.0 \\
(51.0-57.0)\end{array}$ & $\begin{array}{l}53.5 \\
(49.0-49.0)\end{array}$ & $\begin{array}{l}53.5 \\
(49.5-56.8)\end{array}$ & $\begin{array}{l}58.0 \\
(52.0-61.0)\end{array}$ & $\begin{array}{l}56.0 \\
(56.0-56.0)\end{array}$ & $\begin{array}{l}57.0 \\
(53.0-60.3)\end{array}$ & $1.7(1.7-3.2)$ & $7.1(7.1-7.1)$ & $2.5(1.7-5.3)$ \\
\hline Ureter & $1(0.3)$ & $3(1.2)$ & $4(0.7)$ & $\begin{array}{l}56.0 \\
(56.0-56.0)\end{array}$ & $\begin{array}{l}52.5 \\
(48.5-54.5)\end{array}$ & $\begin{array}{l}52.5 \\
(48.8-57.0)\end{array}$ & $\begin{array}{l}61.0 \\
(61.0-61.0)\end{array}$ & $\begin{array}{l}58.0 \\
(56.0-63.5)\end{array}$ & $\begin{array}{l}59.5 \\
(57.0-63.0)\end{array}$ & $5.3(5.3-5.3)$ & \begin{tabular}{|l|}
9.6 \\
$(7.3-10.0)$
\end{tabular} & $7.5(5.2-9.8)$ \\
\hline Oesophagus & $2(0.6)$ & $1(0.4)$ & $3(0.5)$ & \begin{tabular}{|l|}
57.5 \\
$(55.3-59.8)$
\end{tabular} & $\begin{array}{l}58.0 \\
(58.0-58.0)\end{array}$ & $\begin{array}{l}58.0 \\
(55.5-60.0)\end{array}$ & \begin{tabular}{|l|}
67.0 \\
$(66.0-68.0)$
\end{tabular} & $\begin{array}{l}65.0 \\
(65.0-65.0)\end{array}$ & $\begin{array}{l}65.0 \\
(65.0-67.0)\end{array}$ & $\begin{array}{l}9.6 \\
(8.4-10.9)\end{array}$ & $7.1(7.1-7.1)$ & $7.2(7.2-9.6)$ \\
\hline $\begin{array}{l}\text { Central } \\
\text { nervous } \\
\text { system }\end{array}$ & $2(0.6)$ & $0(0.0)$ & $2(0.3)$ & $\begin{array}{l}53.5 \\
(51.3-55.8)\end{array}$ & - & $\begin{array}{l}53.5 \\
(51.3-55.8)\end{array}$ & $\begin{array}{l}55.5 \\
(53.8-57.3)\end{array}$ & - & $\begin{array}{l}55.5 \\
(53.8-57.3)\end{array}$ & $2.3(1.7-2.9)$ & - & $2.3(1.7-2.9)$ \\
\hline Melanoma & $1(0.3)$ & $0(0.0)$ & $1(0.2)$ & $\begin{array}{l}36.0 \\
(36.0-36.0)\end{array}$ & - & $\begin{array}{l}36.0 \\
(36.0-36.0)\end{array}$ & $\begin{array}{l}40.0 \\
(40.0-40.0)\end{array}$ & - & $\begin{array}{l}40.0 \\
(40.0-40.0)\end{array}$ & $4.2(4.2-4.2)$ & - & $4.2(4.2-4.2)$ \\
\hline $\begin{array}{l}\text { Hodgkin } \\
\text { lymphoma }\end{array}$ & $1(0.3)$ & $0(0.0)$ & $1(0.2)$ & $\begin{array}{l}53.0 \\
(53.0-53.0)\end{array}$ & - & $\begin{array}{l}53.0 \\
(53.0-53.0)\end{array}$ & $\begin{array}{l}53.0 \\
(53.0-53.0)\end{array}$ & - & $\begin{array}{l}53.0 \\
(53.0-53.0)\end{array}$ & $0.8(0.8-0.8)$ & - & $0.8(0.8-0.8)$ \\
\hline
\end{tabular}

Table 2. Distribution of post-transplant malignancies. ${ }^{\star}$ Data are expressed as median and interquartile range.

between transplantation and PTM was 4.0 years (interquartile range: 4.8 years). PTM occurred in 49 (8.2\%) recipients within 1 year, $305(51.0 \%)$ between 1 and 5 years, 198 (33.1\%) between 6 and 10 years, and $46(7.7 \%)$ over 10 years. Kaposi sarcoma, gallbladder, oral cavity, and pancreatic cancers developed within 3 years after transplantation, whereas ureter, oesophageal, and NMSC cancers occurred later post-transplant.

The kidney recipients had 3.9 times higher cases of development of any type of cancer when compared with the general Korean population (Table 3). The standardised incidence ratio (SIR) for PTM was higher in female than in male recipients (4.5 vs. 3.3). The SIR of Kaposi sarcoma (192.9), kidney cancer (21.1), and ureter cancer (14.5) were increased more than tenfold while those of gallbladder (1.1), central nervous system (1.2), and oesophageal (1.7) cancers were not significantly higher compared with the general population. In terms of age classes, the SIR of kidney recipients aged less than 40 years showed the highest values for PTMs (16.1 for total PTM, 6.5 for thyroid cancer, 40.0 for colorectal cancer, and 110.8 for kidney cancer) (Supplementary Table S1). 


\begin{tabular}{|c|c|c|c|c|c|c|c|c|c|}
\hline \multirow[b]{2}{*}{ Type of cancer } & \multicolumn{3}{|c|}{$\begin{array}{l}\text { Observed rates of cancer } \\
\text { cases }\end{array}$} & \multicolumn{3}{|c|}{$\begin{array}{l}\text { Expected rates of cancer } \\
\text { cases }^{*}\end{array}$} & \multicolumn{3}{|c|}{ Standardised incidence ratio $(95 \% \mathrm{CI})$} \\
\hline & Male & Female & Total & Male & Female & Total & Male & Female & Total \\
\hline Thyroid & 93.2 & 253.9 & 157.9 & 15.7 & 72.9 & 44.2 & $5.9(4.8-7.2)$ & $3.5(3.1-3.9)$ & $3.6(3.0-4.2)$ \\
\hline Colorectal & 105.7 & 152.4 & 124.5 & 45.4 & 25.2 & 34.2 & $2.3(1.9-2.8)$ & $6.0(5.1-7.1)$ & $3.6(3.0-4.3)$ \\
\hline Kidney & 143.0 & 83.1 & 118.9 & 8.3 & 3.3 & 5.6 & $17.1(14.4-20.1)$ & $25.2(20.1-31.2)$ & $21.1(17.4-25.2)$ \\
\hline Stomach & 96.3 & 101.6 & 98.4 & 62.6 & 26.0 & 42.3 & $1.5(1.2-1.9)$ & $3.9(3.2-4.7)$ & $2.3(1.9-2.8)$ \\
\hline Prostate & 152.3 & 0.0 & 91.0 & 22.0 & - & 9.2 & $6.9(5.9-8.1)$ & - & $9.9(7.9-12.1)$ \\
\hline Liver & 99.5 & 46.2 & 78.0 & 46.8 & 15.7 & 31.3 & $2.1(1.7-2.6)$ & $2.9(2.1-3.9)$ & $2.5(2.0-3.1)$ \\
\hline Lung & 96.3 & 50.8 & 78.0 & 47.9 & 14.6 & 28.5 & $2.0(1.6-2.4)$ & $3.5(2.6-4.6)$ & $2.7(2.2-3.4)$ \\
\hline Breast & 3.1 & 161.6 & 66.9 & 0.2 & 44.6 & 22.5 & $15.5(3.1-43.8)$ & $3.6(3.1-4.2)$ & $3.0(2.3-3.8)$ \\
\hline $\begin{array}{l}\text { Non-Hodgkin } \\
\text { lymphoma }\end{array}$ & 40.4 & 64.6 & 50.2 & 7.6 & 5.2 & 6.4 & $5.3(3.7-7.1)$ & $12.3(9.5-15.7)$ & $7.9(5.8-10.4)$ \\
\hline Urinary bladder & 40.4 & 32.3 & 37.2 & 9.2 & 1.7 & 4.9 & $4.4(3.1-5.9)$ & $19.5(13.2-27.3)$ & $7.6(5.4-10.5)$ \\
\hline Cervix & 0.0 & 64.6 & 26.0 & - & 12.9 & 6.6 & - & $5.0(3.9-6.4)$ & $3.9(2.6-5.8)$ \\
\hline $\begin{array}{l}\text { Non-melanoma } \\
\text { skin }\end{array}$ & 28.0 & 18.5 & 24.1 & 4.4 & 4.0 & 4.2 & $6.4(4.2-9.1)$ & $4.6(2.6-7.1)$ & $5.7(3.6-8.4)$ \\
\hline Oral cavity & 28.0 & 18.5 & 24.1 & 4.3 & 1.7 & 2.9 & $6.6(4.3-9.3)$ & $10.7(6.2-16.4)$ & $8.3(5.3-12.3)$ \\
\hline Pancreas & 28.0 & 9.2 & 20.4 & 8.4 & 5.1 & 6.6 & $3.3(2.2-4.8)$ & $1.8(0.8-3.3)$ & $3.1(1.9-4.7)$ \\
\hline Leukaemia & 18.6 & 23.1 & 20.4 & 5.7 & 4.1 & 4.8 & $3.3(1.9-5.1)$ & $5.6(3.6-8.4)$ & $4.3(2.5-6.4)$ \\
\hline Nasopharyngeal & 24.9 & 9.2 & 18.6 & 6.5 & 1.0 & 3.4 & $3.8(2.4-5.6)$ & $9.6(4.3-17.8)$ & $5.4(3.2-8.5)$ \\
\hline Kaposi sarcoma & 24.9 & 4.6 & 16.7 & 0.1 & 0.0 & 0.1 & $\begin{array}{l}219.4(139.2- \\
320.4)\end{array}$ & - & $192.9(109.9-306.9)$ \\
\hline Ovarian & 0.0 & 36.9 & 14.9 & - & 6.3 & 3.2 & - & $5.9(4.1-8.1)$ & $4.6(2.5-7.5)$ \\
\hline Uterus & 0.0 & 32.3 & 13.0 & - & 5.7 & 2.9 & - & $5.7(3.8-7.9)$ & $4.5(2.4-7.7)$ \\
\hline Gallbladder & 15.5 & 4.6 & 11.1 & 10.1 & 9.8 & 9.9 & $1.5(0.9-2.5)$ & $0.5(0.1-1.1)$ & $1.1(0.6-2.0)$ \\
\hline Ureter & 3.1 & 13.9 & 7.4 & 0.7 & 0.3 & 0.5 & $4.2(0.8-11.7)$ & $44.2(23.3-73.0)$ & $14.5(5.5-28.1)$ \\
\hline Oesophagus & 6.2 & 4.6 & 5.6 & 6.7 & 0.5 & 3.2 & $0.9(0.3-2.0)$ & $9.6(2.8-22.8)$ & $1.7(0.6-3.9)$ \\
\hline $\begin{array}{l}\text { Central nervous } \\
\text { system }\end{array}$ & 6.2 & 0.0 & 3.7 & 3.5 & 2.8 & 3.2 & $1.8(0.6-3.7)$ & - & $1.2(0.3-3.0)$ \\
\hline Melanoma & 3.1 & 0.0 & 1.9 & 0.7 & 0.7 & 0.7 & $4.2(0.8-12.0)$ & - & $2.8(0.2-9.5)$ \\
\hline $\begin{array}{l}\text { Hodgkin lym- } \\
\text { phoma }\end{array}$ & 3.1 & 0.0 & 1.9 & 0.6 & 0.3 & 0.4 & $5.6(1.1-15.8)$ & - & $4.4(0.3-15.3)$ \\
\hline Total & 1059.8 & 1186.5 & 1110.8 & 322.1 & 265.4 & 283.1 & $3.3(3.1-3.5)$ & $4.5(4.2-4.7)$ & $3.9(3.7-4.2)$ \\
\hline
\end{tabular}

Table 3. Standardised incidence ratios according to the type of post-transplant malignancy. CI confidence interval. ${ }^{*}$ The expected rates of cancer cases are based on the general population in Korea, adjusted for age and sex.

Mortality of PTM. Of the 598 recipients with PTM, 89 (14.9\%; 55 males and 34 females) died during the study period. The most common cause of cancer-related death in these patients was liver cancer $(\mathrm{n}=13,14.6 \%)$, followed by lung cancer $(n=12,13.5 \%)$, NHL $(n=11,12.4 \%)$, stomach cancer $(n=8,9.0 \%)$, and colorectal cancer $(n=7,7.9 \%)$. The standardised mortality ratio (SMR) of total recipients with PTM was 1.4 and that of female recipients (1.8) was significantly higher than that of male recipients (1.1) (Table 4). Patients with lymphoma (9.3 for Hodgkin lymphoma and 5.5 for NHL), NMSC (7.0), kidney cancer (5.8), and Kaposi sarcoma (5.6) showed significantly higher SMRs than the general population.

Risk factors for PTM. The risk factors for PTM based on Cox multivariate analysis are shown in Table 5. The risk factors independently related to PTM were older age (40-59 years, hazard ratio $[\mathrm{HR}]=1.70$; $95 \%$ confidence interval $[\mathrm{CI}]=1.41-2.06 ; P<0.001$, and over 60 years, $\mathrm{HR}=2.30 ; 95 \% \mathrm{CI}=1.72-3.06 ; P<0.001)$ and graft failure $(\mathrm{HR}=1.64 ; 95 \% \mathrm{CI}=1.26-2.13 ; P<0.001)$. A non-significant relationship between residuals and time was identified (chi-square $=2.30 ; P=0.682$ ) using the Schoenfeld residuals test to confirm the Cox proportional-hazards assumption (Supplementary Fig. S1). In addition, the Wald chi-square test for goodness of fit was significant $(60.98 ; P<0.001)$, and variance inflation factors ranged from 1.004 to 1.177 which were less than 10 , indicating non-multicollinearity. Figure 1a illustrates the total cumulative incidence of PTM and Fig. 1b,c illustrate the effect of significant factors on cumulative incidence. The estimated cumulative incidence rates of PTM at 1, 2, 5, 10 , and 14 years were $0.54 \%, 1.70 \%, 4.88 \%, 11.23 \%$, and $17.49 \%$ respectively.

\section{Discussion}

In the present study, a comprehensive analysis of PTM after kidney transplantation was conducted based on nationwide data from Korea with a lengthy follow-up duration and information on mortality. The SIR of PTM was 3.9, similar to previous studies ${ }^{11,16,17}$. The SMR of total PTM was 1.4 , and that of lymphoma showed the 


\begin{tabular}{|l|r|r|r|r|l|l|l|l|l|l|}
\hline \multirow{2}{*}{ Type of cancer } & \multicolumn{9}{|l|}{$\begin{array}{l}\text { Observed rates of cancer } \\
\text { death }\end{array}$} & \multicolumn{3}{l}{$\begin{array}{l}\text { Expected rates of cancer } \\
\text { death }\end{array}$} & \multicolumn{2}{l|}{ Standardised mortality ratio (95\% CI) } \\
\cline { 2 - 13 } & Male & Female & Total & Male & Female & Total & Male & Female & Total \\
\hline Liver & 31.1 & 13.9 & 24.1 & 35.0 & 11.4 & 23.1 & $0.9(0.6-1.3)$ & $1.2(0.6-2.0)$ & $1.0(0.7-1.5)$ \\
\hline Lung & 31.1 & 9.2 & 22.3 & 47.5 & 17.0 & 32.3 & $0.7(0.4-0.9)$ & $0.5(0.2-1.0)$ & $0.7(0.4-1.0)$ \\
\hline Non-Hodgkin lymphoma & 15.5 & 27.7 & 20.4 & 4.1 & 3.2 & 3.7 & $3.8(2.1-6.2)$ & $8.7(5.7-12.5)$ & $5.5(3.3-8.3)$ \\
\hline Stomach & 21.8 & 4.6 & 14.9 & 26.5 & 14.3 & 20.4 & $0.8(0.5-1.2)$ & $0.3(0.1-0.8)$ & $0.7(0.4-1.2)$ \\
\hline Colorectal & 9.3 & 18.5 & 13.0 & 5.6 & 4.3 & 4.9 & $1.7(0.7-3.1)$ & $4.3(2.5-6.6)$ & $2.7(1.4-4.5)$ \\
\hline Kidney & 6.2 & 13.9 & 9.3 & 2.2 & 0.9 & 1.6 & $2.8(1.0-5.9)$ & $15.4(8.1-25.4)$ & $5.8(2.6-10.7)$ \\
\hline Nasopharyngeal & 12.4 & 0.0 & 7.4 & 3.1 & 0.9 & 2.0 & $4.0(2.0-6.8)$ & & $3.7(1.4-7.2)$ \\
\hline Urinary bladder & 6.2 & 4.6 & 5.6 & 3.3 & 1.1 & 2.2 & $1.9(0.7-4.0)$ & $4.2(1.2-10.0)$ & $2.5(0.9-5.6)$ \\
\hline Ovarian & 0.0 & 13.9 & 5.6 & 0.0 & 3.5 & 1.7 & & $4.0(2.1-6.5)$ & $3.3(1.1-7.3)$ \\
\hline Prostate & 9.3 & 0.0 & 5.6 & 5.0 & 0.0 & 2.5 & $1.9(0.8-3.4)$ & & $2.2(0.8-4.9)$ \\
\hline Non-melanoma skin & 3.1 & 9.2 & 5.6 & 0.8 & 0.8 & 0.8 & $3.9(0.8-11.0)$ & $11.5(5.1-21.4)$ & $7.0(2.4-15.5)$ \\
\hline Breast & 0.0 & 13.9 & 5.6 & 0.1 & 7.5 & 3.8 & 0.0 & $1.8(1.0-3.0)$ & $1.5(0.5-3.3)$ \\
\hline Kaposi sarcoma & 6.2 & 4.6 & 5.6 & 1.0 & 0.9 & 1.0 & $6.2(2.2-13.1)$ & $5.1(1.5-12.2)$ & $5.6(1.9-12.4)$ \\
\hline Pancreas & 6.2 & 0.0 & 3.7 & 9.0 & 8.0 & 8.0 & $0.7(0.2-1.5)$ & & $0.5(0.1-1.2)$ \\
\hline Central nervous system & 6.2 & 0.0 & 3.7 & 2.6 & 2.2 & 2.4 & $2.4(0.8-5.0)$ & & $1.5(0.4-4.0)$ \\
\hline Cervix & 0.0 & 9.2 & 3.7 & 0.0 & 4.0 & 2.0 & & $2.3(1.0-4.3)$ & $1.9(0.4-4.8)$ \\
\hline Thyroid & 0.0 & 4.6 & 1.9 & 0.4 & 1.0 & 0.7 & & $4.6(1.4-11.0)$ & $2.7(0.2-9.2)$ \\
\hline Leukaemia & 0.0 & 4.6 & 1.9 & 4.1 & 3.1 & 3.6 & & $1.5(0.4-3.5)$ & $0.5(0.0-1.8)$ \\
\hline Oesophagus & 3.1 & 0.0 & 1.9 & 5.4 & 0.5 & 3.0 & $0.6(0.1-1.6)$ & & $0.6(0.0-2.1)$ \\
\hline Ureter & 0.0 & 4.6 & 1.9 & 0.7 & 0.5 & 0.6 & & $9.2(2.7-21.9)$ & $3.1(0.2-10.7)$ \\
\hline Hodgkin lymphoma & 3.1 & 0.0 & 1.9 & 0.3 & 0.2 & 0.2 & $10.4(2.1-29.2)$ & & $9.3(0.5-32.1)$ \\
\hline Total & 170.9 & 157.0 & 165.3 & 156.7 & 85.3 & 120.5 & $1.1(0.9-1.3)$ & $1.8(1.6-2.1)$ & $1.4(1.2-1.6)$ \\
\hline
\end{tabular}

Table 4. Standardised mortality ratios according to the types of post-transplant malignancies. CI confidence interval. ${ }^{*}$ The expected rates of cancer death are based on the general population in Korea, adjusted for age and sex.

\begin{tabular}{|c|c|c|c|c|}
\hline \multirow[b]{2}{*}{ Variable } & \multicolumn{2}{|c|}{ Univariate for PTM } & \multicolumn{2}{|c|}{ Multivariate for PTM* } \\
\hline & HR (95\% CI) & $P$-value & HR $(95 \% \mathrm{CI})$ & $P$-value \\
\hline \multicolumn{5}{|l|}{ Age, years } \\
\hline$<40$ & Reference & & Reference & \\
\hline $40-59$ & $1.70(1.41-2.06)$ & $<0.001$ & $1.70(1.41-2.06)$ & $<0.001$ \\
\hline$>59$ & $2.29(1.72-3.05)$ & $<0.001$ & $2.30(1.72-3.06)$ & $<0.001$ \\
\hline \multicolumn{5}{|l|}{ Sex } \\
\hline Male & Reference & & Reference & \\
\hline Female & $1.12(0.95-1.32)$ & 0.166 & & \\
\hline \multicolumn{5}{|l|}{ Induction immunosuppressant } \\
\hline Basiliximab & $1.15(0.86-1.56)$ & 0.348 & & \\
\hline Anti-thymocyte globulin & $1.26(1.04-1.52)$ & 0.017 & $1.20(0.99-1.46)$ & 0.058 \\
\hline \multicolumn{5}{|l|}{ Maintenance immunosuppressant } \\
\hline Tacrolimus + MMF + corticosteroid & $1.14(0.96-1.34)$ & 0.135 & & \\
\hline Cyclosporine + MMF + corticosteroid & $1.03(0.87-1.23)$ & 0.715 & & \\
\hline \multicolumn{5}{|l|}{ Infection } \\
\hline CMV disease & $1.04(0.80-1.35)$ & 0.788 & & \\
\hline EBV mononucleosis & $1.10(0.15-7.79)$ & 0.928 & & \\
\hline Graft failure & $1.56(1.20-2.02)$ & 0.001 & $1.64(1.26-2.13)$ & $<0.001$ \\
\hline \multicolumn{5}{|l|}{ Year of transplant } \\
\hline $2003-2009$ & Reference & & Reference & \\
\hline 2010-2016 & $1.13(0.94-1.39)$ & 0.193 & & \\
\hline
\end{tabular}

Table 5. Univariate and multivariate analysis for post-transplant malignancies. $C M V$ cytomegalovirus, $E B V$ Epstein-Barr virus, $M M F$ mycophenolate mofetil, $H R$ hazard ratio, PTM post-transplant malignancy. *Variables less than 0.05 of $P$-values in univariate analysis were included in the multivariate analysis. 

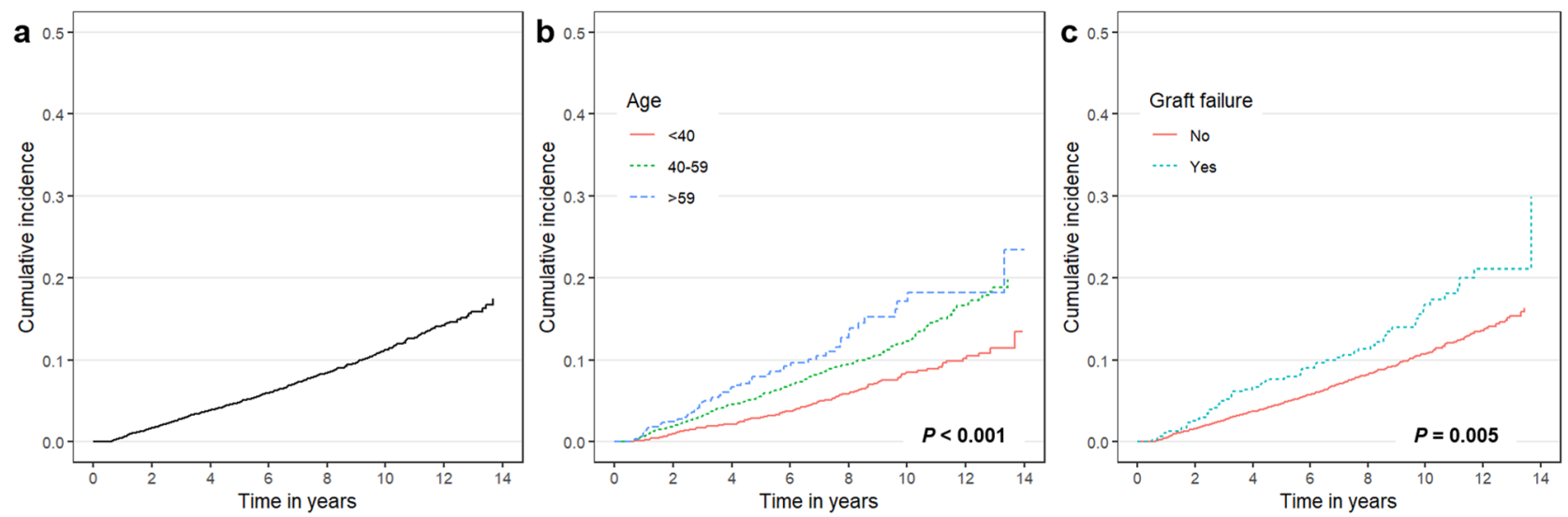

Figure 1. Cumulative incidence rate of post-transplant malignancy after kidney transplantation. (a) Total incidence. (b) Older age and (c) graft failure were associated with post-transplant malignancy.

highest value (9.3 for Hodgkin lymphoma and 5.5 for NHL). Older age and graft failure were significant risk factors for PTM.

Kidney recipients have higher cases of developing cancer (SIR, 1.7-3.9) compared with the general population ${ }^{7-12,20-22}$. The incidence of PTM varies according to country and type of malignancy. In general,

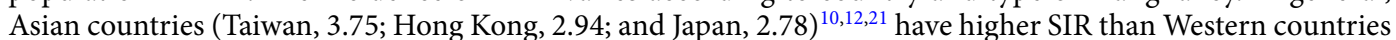

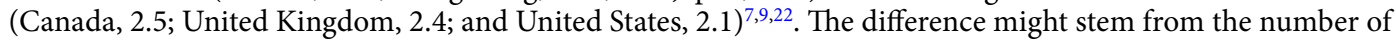
included patients, hospital- or population-based designs, and the epoch of transplantation.

Regarding the types of PTM, the most common malignancy was thyroid cancer, followed by colorectal, kidney, stomach, and prostate cancer. The high frequency of these cancers demonstrated previously ${ }^{16,17}$ may result from increased surveillance.

When compared with the general population, the cases of Kaposi sarcoma were the most prominent with an SIR of 192.9 despite a small number of incident cases. An immunocompromised state after kidney transplantation could increase the virus infection that plays an important role in the pathology of Kaposi sarcoma ${ }^{23}$. Further, the lower incidence of Kaposi sarcoma on Korean general population ${ }^{24}$ could have caused the substantially high SIR seen in this study. In terms of kidney cancer having high frequency and SIR, our results were consistent with those of previous studies ${ }^{21,25}$. Although the exact mechanism has not been revealed, malignant transformation of cysts developed during kidney failure, the duration of dialysis, and an immunocompromised state due to nephrotoxic drugs might be the cause of these high kidney cancer values ${ }^{21,26}$.

Regarding mortality associated with PTM, only a few population-based studies have dealt with this subject. According to a recently published Australian and New Zealand population-based cohort study, $20 \%$ of cancer deaths were reported, which is slightly higher than our results $(14.9 \%)^{19}$. The most common cause of cancer death was lung cancer (21\%), followed by liver cancer (18\%) and NHL (16\%). This rank is similar to our results ( $14.6 \%$ for liver cancers, $13.5 \%$ for lung, and $12.4 \%$ for NHL). Smoking is a well-known risk factor for lung cancer ${ }^{27}$ and hepatitis B virus is one of the established risks of liver cancer ${ }^{16,28}$. In particular, South Korea is an epidemic region for this hepatitis virus. Virus vaccination, prohibitions against smoking, and low dose-computed tomography should be recommended for recipients. In addition, the reduction of spicy food intake, eradication of Helicobacter pylori, and screening with gastroscopy for stomach cancer ( $9.0 \%$ of cause of death), which still shows a higher proportion of PTM in Asia ${ }^{11,12}$, should be encouraged.

In terms of SMR, the Australian and New Zealand population-based cohort study reported an SMR of 2.9 for all-site cancers ${ }^{19}$. In the Asian population, a similar SMR value of 2.3 was reported based on the Hong Kong Renal Registry ${ }^{21}$. A population-based cohort study in Canada found an SMR of 2.8; however, the SMR decreased to 1.9 when recipients with cancers before transplantation were excluded ${ }^{18}$. In this study, we presented the SMR for Korea and found a lower SMR (1.4) than the SMRs for Australian and New Zealand, and Hong Kong studies. However, the SMR was still significantly elevated, similar to the previous Canadian study. The exclusion of recipients with pre-transplant malignancies is likely the cause of these results.

The cancer type with the highest SMR was lymphoma ( 9.3 for Hodgkin lymphoma and 5.5 for NHL). When compared with our results, higher SMR values have been reported in previous studies (42.2 for Australian and New Zealand; 18.2 for Hong Kong; and 14.1 for Italy) $)^{6,19,21}$. These different SMR values might be attributed to the higher SIRs of lymphoma in previous studies mostly conducted in Western countries.

Multivariate analysis revealed that the recipient's age at transplantation and graft failure were significant risk factors for development of PTM. Concordant with our results, the recipient's age has been widely reported to be a risk factor for $\mathrm{PTM}^{11}$. The close relationship between graft failure and development of cancers has also been confirmed in previously reported studies ${ }^{29}$. Immunosuppression associated with graft failure might predispose to the development of PTM. Therefore, alternative regimens to current immunosuppressants such as mammalian target of rapamycin inhibitors that may lead to better outcomes regarding PTM have been investigated ${ }^{29,30}$. In South Korea, most recipients are on a triple therapy regimen that includes a calcineurin inhibitor, mycophenolic acid, and steroids as the major initial maintenance immunosuppressants $(81.4 \%)^{31}$. Among calcineurin 
inhibitors, tacrolimus is prescribed far more than cyclosporine, which was reported to be associated with carcinogenic effects ${ }^{32}$. Regarding level, a randomised comparison study demonstrated that recipients with a lower level of cyclosporine had a lower incidence of PTM than those with a normal level ${ }^{33}$. The effects of combination of immunosuppressant, and dosing of them for PTM according to cancer types are still controversial ${ }^{29}$, thus further studies are necessary.

This study had several limitations. The lack of detailed clinical information such as type of pathology, laboratory data, smoking, drinking, donor status, immunologic profiles, family history, dosage of differently combined immunosuppressive drugs, and target drug levels led to restrictions on the analysis of PTM. Moreover, inevitable classification bias could exist because we used registry data based on physicians' diagnoses. Furthermore, fatal cancers such as early post-transplant lymphoproliferative disorder ${ }^{34}$, which could develop within 6 months after kidney transplantation would be omitted because of predefined criteria based on a previous study investigating de novo non-cutaneous $\mathrm{PTM}^{5}$. The precise identification and calculation of SIR and SMR for multiple or recurrent malignancies were also difficult in this type of dataset. Therefore, we considered the first malignancy as PTM due to the strong effect of de novo malignancy on mortality. Further studies focusing on early-onset PTM, multiple and recurrent malignancies, and specific risk factors for PTM are necessary. Hospital-based designs that include participating multi-centres for precise identification of variables and a large study population would complement our limits in the present study. Despite these limitations, the strength of this study is the use of a nationwide population database of kidney recipients with 14 years of lengthy follow-up for late-onset malignancies. Studies handling PTM with SMR using a nationwide data source are rare, particularly in Asia. The relatively large sample size of the entire national population and unbiased measures used in this study could provide reliable information about PTM in kidney recipients.

In conclusion, our study presented a comprehensive analysis of PTM after kidney transplantation, including SIR, SMR, and risk factors. We found that the SIR of malignancy in kidney transplant recipients was 3.9, and provided information about late-onset PTM within 14 years, which might be useful for long-lived recipients. Furthermore, this study is the first to reveal that the SMR of PTM is slightly higher than that in the general population in South Korea. Although increases in cancer incidence of certain types of PTM translate into similar increases in cancer mortality, fatal malignancies significantly related to mortality such as liver and lung cancers, lymphoma, and stomach cancer need more intensive care based on data used in this study. Further, risk factors for PTM including older age and graft failure should be considered when managing transplant recipients. These findings reflecting geographical variation can facilitate the development of cancer prevention strategies and follow-up recommendations for the improvement of the outcome of kidney recipients.

\section{Methods}

Study design. This was a retrospective and observational cohort study that used prospectively registered national data sets for reimbursement purposes. All patients who underwent kidney transplantation procedures (Z94.0 code of the International Classification of Disease, 10th revision, Clinical Modification [ICD-10-CM]) at any Korean medical centre from January 2003 to December 2016 were included. We included first malignancy after kidney transplantation as PTM because the outcome of multiple or recurrent cancers could be affected by the type of first malignancy. De novo malignancy exhibited a more aggressive tendency, and was one of the leading causes of mortality according to previous studies ${ }^{35,36}$. The recipients with pre-transplant cancers were excluded in order to focus on de novo malignancy ${ }^{18}$. Cancer cases that occurred within 6 months after kidney transplantation were excluded from analysis due to the possibility of undiagnosed malignancies before surgery based on the criteria of a previous study for de novo non-cutaneous $\mathrm{PTM}^{5}$. Malignancies detected after graft failure were also excluded. We investigated the incidence, mortality, and risk factors related to PTM.

This study was approved by the independent Institutional Review Board of Kosin University Gospel Hospital (KUGH 2017-12-009). The data acquisition number for the National Health Insurance Sharing Service was REQ0000019170. This study was conducted in accordance with the Declaration of Helsinki. The need for informed consent was waived because anonymity of personal information was maintained.

Study population (patient selection). The study included all patients who had been listed for kidney transplantation from January 2003 to December 2016 in the Health Insurance Review and Assessment Service (HIRA). The patients were registered in the HIRA database after kidney transplantation, as defined by the ICD10-CM code Z94.0. During this period, 18,822 patients were enrolled in the database. We excluded 6089 patients who were not diagnosed with cancer as the main disease, and registered in the reimbursement program for cancer patients properly. Additionally, 1898 patients who were diagnosed with cancer before transplantation, and 430 patients who were diagnosed with cancer within 6 months after kidney transplantation or after graft failure were not included. Patients under 20 years $(n=410)$ and 53 patients who underwent other organ transplantations were also excluded. Twenty-seven recipients with missing variables such as age, and sex were not included. The final cohort consisted of 9915 patients and included 598 patients with PTM (Supplementary Fig. S2). The records of medical visits, demographic characteristics, and death status were collected from the HIRA database for all kidney recipients with and without PTM.

Study variables. We collected the following demographic data and baseline characteristics of kidney recipients from the HIRA database: age, sex, immunosuppressive agents, the presence of cytomegalovirus (CMV) disease, Epstein-Barr virus (EBV) mononucleosis, year of transplantation, cancer type, and date of mortality. CMV infection included CMV diseases (mononucleosis, pneumonitis, hepatitis, and pancreatitis) and the posttransplant administration of antiviral agent (ganciclovir or valganciclovir ${ }^{37}$. The ICD-10-CM codes for CMV 
disease were B27.1, B25.0, B25.1, B25.8, and B25.9. In terms of EBV status, the ICD-10-CM code B27.0 was used. We defined cases of more than 10 dialysis sessions after 90 days post kidney transplantation as graft failure ${ }^{38}$.

Data source. The data used in this study were obtained from the HIRA database, which is based on the NHI system operated by the Korean government. Healthcare institutions submit the medical data for all inpatients and outpatients in electronic format to the HIRA for reimbursement purposes. The claims data integrated by HIRA include all healthcare utilisation information on inpatients and outpatients. Data about the demographic characteristics of the patients, principal diagnosis, prescription history, and performed procedures based on ICD-10-CM codes are included in this database. In this study, we obtained all data about kidney recipients from the RID program of the HIRA database who were registered between January 2003 and the end of December 2016. The Korean government assigned kidney transplantation to the RID system to reduce patient payments. To ensure the quality of registration in the RID program, diagnosis must be based on the uniform criteria provided by the NHI and must be reviewed by the corresponding healthcare institution before submission to the NHI, which confirms the medical record of each registered individual. Therefore, the data registered in the RID registry that are linked with the national health insurance system are verified and reliable ${ }^{39,40}$.

Causes of death for the deceased kidney recipients were also analysed by linking the Statistics Korea data with the HIRA database. In the Statistics Korea data, the causes of death are documented according to the ICD-10 codes, which are verified by physicians at the time of death, and information regarding all casualties was included. Cancer incidence and mortality of the general population were also obtained from the Statistics Korea data.

Statistical analysis. We evaluated the incidence, mortality, and risk factors for PTM. Descriptive statistics were used for patient characteristics associated with PTM. Comparisons of nominal and continuous variables between groups were assessed using the Chi-square and Mann-Whitney $U$ tests, respectively. SIR was calculated as the number of observed PTM cases divided by the expected number of malignancies based on the personyears at risk and the cancer incidence rates in the general population. The SMR was calculated as the observed rates divided by the expected rates of mortality in order to compare with the general population ${ }^{21}$. The PTM related mortality was defined as primary cause of death due to $\mathrm{PTM}^{21}$, and was analysed based on the data provided by Statistics Korea. Multivariate Cox proportional hazards regression models were used to examine the variables having $P$ values less than 0.05 , which were considered to be statistically significant based on univariate analysis $^{41,42}$ for PTM.

Statistical analyses were performed using R statistical software (version 3.4.4; R Foundation for Statistical Computing, Vienna, Austria) and SAS statistical analysis software (version 9.4; SAS Institute Inc., Cary, NC, USA). $P$ values less than 0.05 were considered statistically significant.

\section{Data availability}

All data generated or analysed during this study are included in this published article (Tables and Figures) and available from the corresponding author on reasonable request. The additional raw data are available on request to the National Health Insurance Service, Korea.

Received: 11 February 2020; Accepted: 5 November 2020

Published online: 08 December 2020

\section{References}

1. Au, E., Wong, G. \& Chapman, J. R. Cancer in kidney transplant recipients. Nat. Rev. Nephrol. 14, 508-520. https://doi.org/10.1038/ s41581-018-0022-6 (2018).

2. AlBugami, M. \& Kiberd, B. Malignancies: pre and post transplantation strategies. Transpl. Rev. (Orlando) 28, 76-83. https://doi. org/10.1016/j.trre.2013.12.002 (2014).

3. Kiberd, B. A., Rose, C. \& Gill, J. S. Cancer mortality in kidney transplantation. Am. J. Transpl. 9, 1868-1875. https://doi.org/10.1 $111 / j .1600-6143.2009 .02728 . x(2009)$.

4. Wong, G., Chapman, J. R. \& Craig, J. C. Death from cancer: a sobering truth for patients with kidney transplants. Kidney Int. 85, 1262-1264. https://doi.org/10.1038/ki.2013.494 (2014).

5. Cena, T. et al. De novo noncutaneous malignancies after kidney transplantation are associated with an increased risk of graft failure: results from a time-dependent analysis on 672 patients. Transpl. Int. 29, 1085-1093. https://doi.org/10.1111/tri.12813 (2016).

6. Tessari, G. et al. Incidence of primary and second cancers in renal transplant recipients: a multicenter cohort study. Am. J. Transpl. 13, 214-221. https://doi.org/10.1111/j.1600-6143.2012.04294.x (2013).

7. Engels, E. A. et al. Spectrum of cancer risk among US solid organ transplant recipients. JAMA 306, 1891-1901. https://doi. org/10.1001/jama.2011.1592 (2011).

8. Adami, J. et al. Cancer risk following organ transplantation: a nationwide cohort study in Sweden. Br. J. Cancer 89, $1221-1227$. https://doi.org/10.1038/sj.bjc.6601219 (2003).

9. Collett, D., Mumford, L., Banner, N. R., Neuberger, J. \& Watson, C. Comparison of the incidence of malignancy in recipients of different types of organ: a UK Registry audit. Am. J. Transpl. 10, 1889-1896. https://doi.org/10.1111/j.1600-6143.2010.03181.x (2010).

10. Li, W. H. et al. Malignancies after renal transplantation in Taiwan: a nationwide population-based study. Nephrol. Dial. Transpl. 27, 833-839. https://doi.org/10.1093/ndt/gfr277 (2012).

11. Hwang, J. K., Moon, I. S. \& Kim, J. I. Malignancies after kidney transplantation: a 40-year single-center experience in Korea. Transpl. Int. 24, 716-721. https://doi.org/10.1111/j.1432-2277.2011.01270.x (2011).

12. Hoshida, Y. et al. Cancer risk after renal transplantation in Japan. Int. J. Cancer 71, 517-520 (1997).

13. Kim, J. H., Kim, S. O., Han, D. J. \& Park, S. K. Post-transplant malignancy: a burdensome complication in renal allograft recipients in Korea. Clin. Transpl. 28, 434-442. https://doi.org/10.1111/ctr.12328 (2014).

14. Ju, M. K. et al. Chronologically different incidences of post-transplant malignancies in renal transplant recipients: single center experience. Transpl. Int. 22, 644-653. https://doi.org/10.1111/j.1432-2277.2009.00846.x (2009).

15. Min, S. K. et al. Malignancy in renal transplant recipients. Transpl. Proc. 32, 1980-1981 (2000). 
16. Heo, J., Noh, O. K., Oh, Y. T., Chun, M. \& Kim, L. Cancer risk after renal transplantation in South Korea: a nationwide populationbased study. BMC Nephrol. 19, 311. https://doi.org/10.1186/s12882-018-1110-3 (2018).

17. Park, B. et al. De novo cancer incidence after kidney and liver transplantation: results from a nationwide population based data. Sci. Rep. 9, 17202. https://doi.org/10.1038/s41598-019-53163-9 (2019).

18. Acuna, S. A. et al. Cancer mortality among recipients of solid-organ transplantation in Ontario, Canada. JAMA Oncol. 2, 463-469. https://doi.org/10.1001/jamaoncol.2015.5137 (2016).

19. Rosales, B. M. et al. Cancer mortality in kidney transplant recipients: an Australian and New Zealand population-based cohort study, 1980-2013. Int. J. Cancer. https://doi.org/10.1002/ijc.32585 (2019).

20. Piselli, P. et al. Risk of de novo cancers after transplantation: results from a cohort of 7217 kidney transplant recipients, Italy 1997-2009. Eur. J. Cancer 49, 336-344. https://doi.org/10.1016/j.ejca.2012.09.013 (2013).

21. Cheung, C. Y. et al. Malignancies after kidney transplantation: Hong Kong renal registry. Am. J. Transpl. 12, 3039-3046. https:// doi.org/10.1111/j.1600-6143.2012.04209.x (2012).

22. Villeneuve, P. J. et al. Cancer incidence among Canadian kidney transplant recipients. Am. J. Transpl. 7, 941-948. https://doi.org /10.1111/j.1600-6143.2007.01736.x (2007).

23. Frances, C. et al. The impact of preexisting or acquired Kaposi sarcoma herpesvirus infection in kidney transplant recipients on morbidity and survival. Am. J. Transpl. 9, 2580-2586. https://doi.org/10.1111/j.1600-6143.2009.02816.x (2009).

24. de Sanjose, S. et al. Geographic variation in the prevalence of Kaposi sarcoma-associated herpesvirus and risk factors for transmission. J. Infect. Dis. 199, 1449-1456. https://doi.org/10.1086/598523 (2009).

25. Wang, Y., Lan, G. B., Peng, F. H. \& Xie, X. B. Cancer risks in recipients of renal transplants: a meta-analysis of cohort studies. Oncotarget 9, 15375-15385. https://doi.org/10.18632/oncotarget.23841 (2018).

26. Shapiro, R. \& Kaplan, B. Native renal cysts and dialysis duration are risk factors for renal cell carcinoma in renal transplant recipients. Am. J. Transpl. 11, 10. https://doi.org/10.1111/j.1600-6143.2010.03376.x (2011).

27. Khalil, M. A. M. et al. Cigarette smoking and its hazards in kidney transplantation. Adv. Med. 2017, 6213814. https://doi. org/10.1155/2017/6213814 (2017).

28. Marinaki, S., Kolovou, K., Sakellariou, S., Boletis, J. N. \& Delladetsima, I. K. Hepatitis B in renal transplant patients. World J. Hepatol. 9, 1054-1063. https://doi.org/10.4254/wjh.v9.i25.1054 (2017).

29. Sprangers, B., Nair, V., Launay-Vacher, V., Riella, L. V. \& Jhaveri, K. D. Risk factors associated with post-kidney transplant malignancies: an article from the Cancer-Kidney International Network. Clin. Kidney J. 11, 315-329. https://doi.org/10.1093/ckj/sfx12 2 (2018).

30. van Leeuwen, M. T. et al. Effect of reduced immunosuppression after kidney transplant failure on risk of cancer: population based retrospective cohort study. BMJ 340, c570. https://doi.org/10.1136/bmj.c570 (2010).

31. Chang, J. Y. et al. Immunosuppressant prescription pattern and trend in kidney transplantation: a multicenter study in Korea. PLoS ONE 12, e0183826. https://doi.org/10.1371/journal.pone.0183826 (2017).

32. Lim, W. H. et al. The risk of cancer in kidney transplant recipients may be reduced in those maintained on everolimus and reduced cyclosporine. Kidney Int. 91, 954-963. https://doi.org/10.1016/j.kint.2016.11.008 (2017).

33. Dantal, J. et al. Effect of long-term immunosuppression in kidney-graft recipients on cancer incidence: randomised comparison of two cyclosporin regimens. Lancet 351, 623-628. https://doi.org/10.1016/S0140-6736(97)08496-1 (1998).

34. Cheung, C. Y., Ma, M. K. M., Chau, K. F., Chak, W. L. \& Tang, S. C. W. Posttransplant lymphoproliferative disorders in kidney transplant recipients: a retrospective cohort analysis over two decades in Hong Kong. Oncotarget 8, 96903-96912. https://doi. org/10.18632/oncotarget.18890 (2017).

35. Doycheva, I., Amer, S. \& De Watt, K. D. Novo malignancies after transplantation: risk and surveillance strategies. Med. Clin. N. Am. 100, 551-567. https://doi.org/10.1016/j.mcna.2016.01.006 (2016).

36. McCaughan, J. A. \& Courtney, A. E. The clinical course of kidney transplant recipients after 20 years of graft function. Am. J. Transpl. 15, 734-740. https://doi.org/10.1111/ajt.13041 (2015).

37. Azevedo, L. S. et al. Cytomegalovirus infection in transplant recipients. Clinics 70, 515-523. https://doi.org/10.6061/clini cs/2015(07)09 (2015).

38. Farrugia, D. et al. Death within the first year after kidney transplantation-an observational cohort study. Transpl. Int. 27, 262-270. https://doi.org/10.1111/tri.12218 (2014).

39. Ahn, I. M. et al. Incidence, prevalence, and survival of moyamoya disease in Korea: a nationwide, population-based study. Stroke 45, 1090-1095. https://doi.org/10.1161/STROKEAHA.113.004273 (2014).

40. Yeom, M. S. Direction of policy and management for rare intractable disease patients support program [in Korean]. Health Insur. Rev. Assess. Serv. (HIRA) Policy Rev. 3, 6 (2009).

41. Choi, H. et al. The risk factors associated with treatment-related mortality in 16,073 kidney transplantation-a nationwide cohort study. PLoS ONE 15, e0236274. https://doi.org/10.1371/journal.pone.0236274 (2020).

42. Jeong, S. et al. Genetic heterogeneity and prognostic impact of recurrent ANK2 and TP53 mutations in mantle cell lymphoma: a multi-centre cohort study. Sci. Rep. 10, 13359. https://doi.org/10.1038/s41598-020-70310-9 (2020).

\section{Acknowledgements}

The authors are grateful to Hyun Jung Kim and Hyeong Sik Ahn and the staff of the Department of Preventive Medicine, College of Medicine, Korea University, for their assistance in preparing this article. This work was supported by the National Research Foundation of Korea (NRF) grant, funded by the Korean government (Ministry of Science and ICT) [grant number NRF-2017R1C1B2004597].

\section{Author contributions}

S.J., S.L., and H.J.K. designed the study and were major contributors in writing the manuscript. M.P., W.S., and J.H.R. made substantial contributions to development of the study design and analysis. H.S.L., S.G.K., and D.J.K. led the data collection and conducted the data analysis. All authors read and approved the manuscript.

\section{Competing interests}

The authors declare no competing interests.

\section{Additional information}

Supplementary information is available for this paper at https://doi.org/10.1038/s41598-020-78283-5.

Correspondence and requests for materials should be addressed to H.J.K.

Reprints and permissions information is available at www.nature.com/reprints. 
Publisher's note Springer Nature remains neutral with regard to jurisdictional claims in published maps and institutional affiliations.

(c) (i) Open Access This article is licensed under a Creative Commons Attribution 4.0 International License, which permits use, sharing, adaptation, distribution and reproduction in any medium or format, as long as you give appropriate credit to the original author(s) and the source, provide a link to the Creative Commons licence, and indicate if changes were made. The images or other third party material in this article are included in the article's Creative Commons licence, unless indicated otherwise in a credit line to the material. If material is not included in the article's Creative Commons licence and your intended use is not permitted by statutory regulation or exceeds the permitted use, you will need to obtain permission directly from the copyright holder. To view a copy of this licence, visit http://creativecommons.org/licenses/by/4.0/.

(c) The Author(s) 2020 